\title{
Activating $\mathrm{MoS}_{2}$ with Super-High Nitrogen-Doping \\ Concentration as Efficient Catalyst for Hydrogen \\ Evolution Reaction
}

Qian Yang,,$+\neq$ Zegao Wang,, \# Lichun Dong,, , s," Wenbin Zhao, ${ }^{+}$Yan Jin, + Liang Fang, ${ }^{\perp}$

Baoshan Hu, ,+ Mingdong Dong,,

tSchool of Chemistry and Chemical Engineering, Chongqing University, Chongqing

401331, China

\#Interdisciplinary Nanoscience Center (iNANO), Aarhus University, DK-8000 Aarhus C,

Denmark

\#School of Materials Science and Engineering, Sichuan University, Chengdu 610065,

PR China 
$\S$ School of Chemistry and Chemical Engineering, Yangtze Normal University, Fuling 408100, Chongqing, China

" Key Laboratory of Low-grade Energy Utilization Technologies \& Systems of the Ministry of Education, Chongqing University, Chongqing 40004, China

${ }^{\perp}$ College of Physics, Chongqing University, Chongqing 401331, China
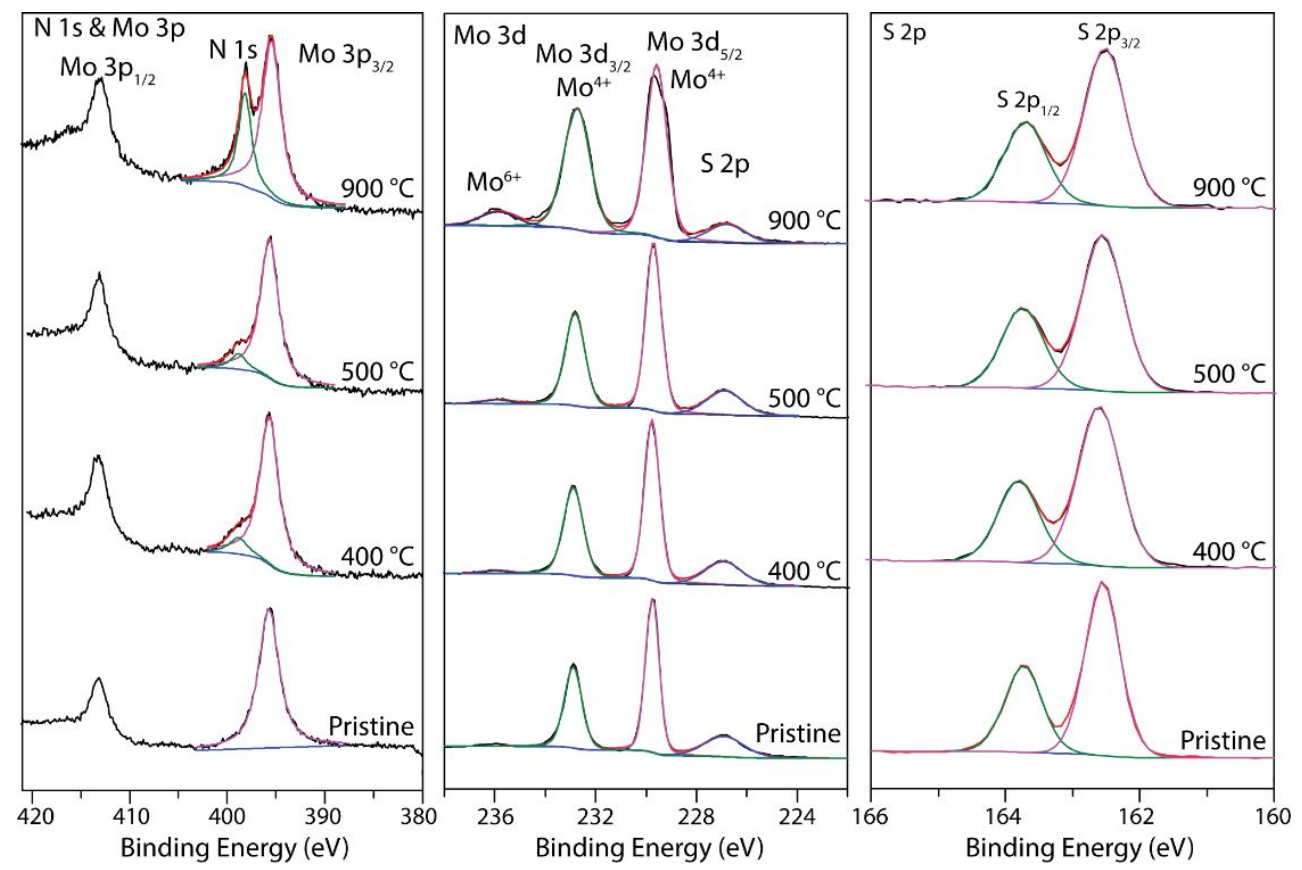

Figure S1. High resolution XPS spectra of N 1s, Mo 3d, and S 2p of pristine $M_{0} S_{2}$ and $\mathrm{N}-\mathrm{MoS}_{2}$ with different activation temperatures. 

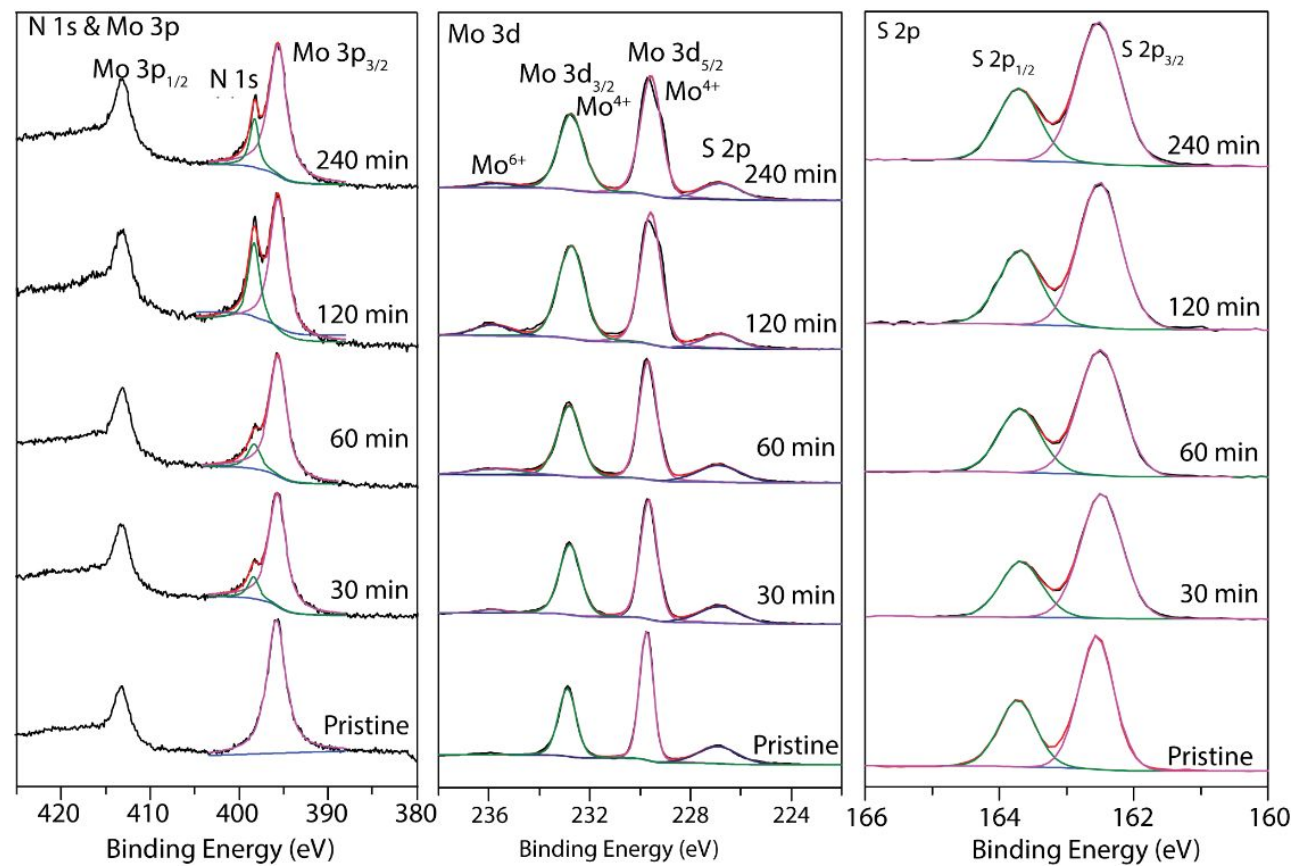

Figure S2. High resolution XPS spectra of N 1s, Mo 3d, and S 2p of pristine $\mathrm{MoS}_{2}$ and

$\mathrm{N}-\mathrm{MoS}_{2}$ with different activation times at $900{ }^{\circ} \mathrm{C}$. 


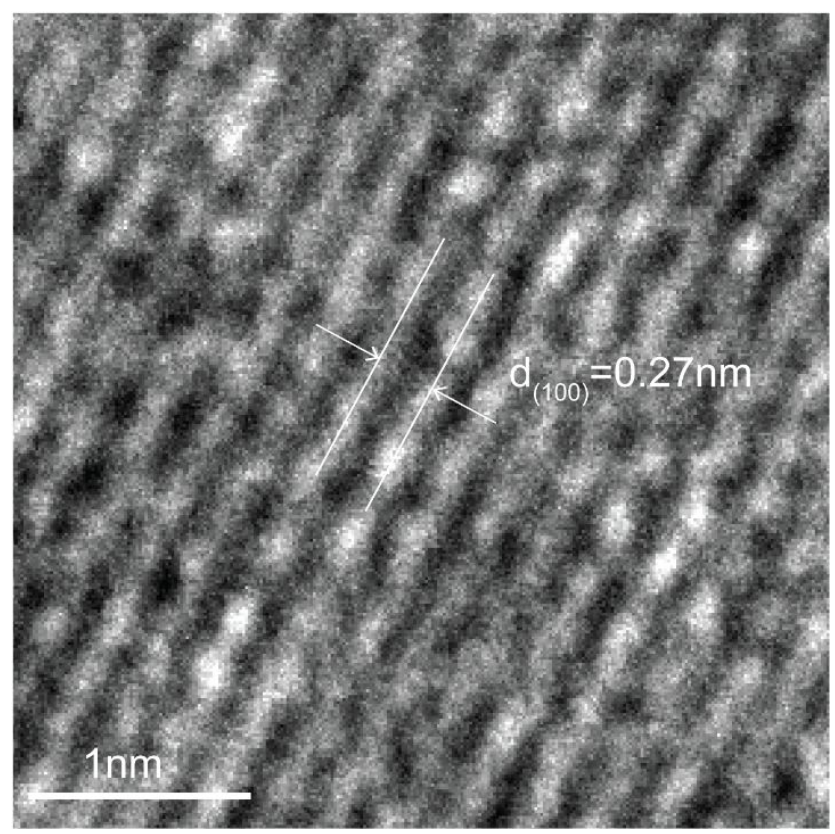

Figure S3. HR-TEM image of pristine $\mathrm{MoS}_{2}$.

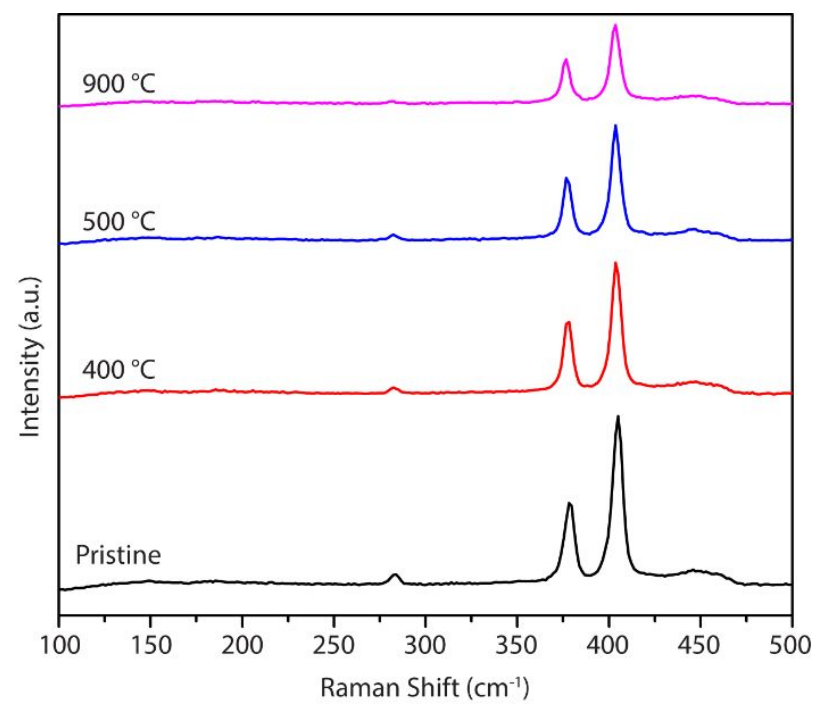

Figure S4. Raman spectra of pristine $\mathrm{MoS}_{2}$ and $\mathrm{N}-\mathrm{MoS}_{2}$ treated at different activation

temperatures. 

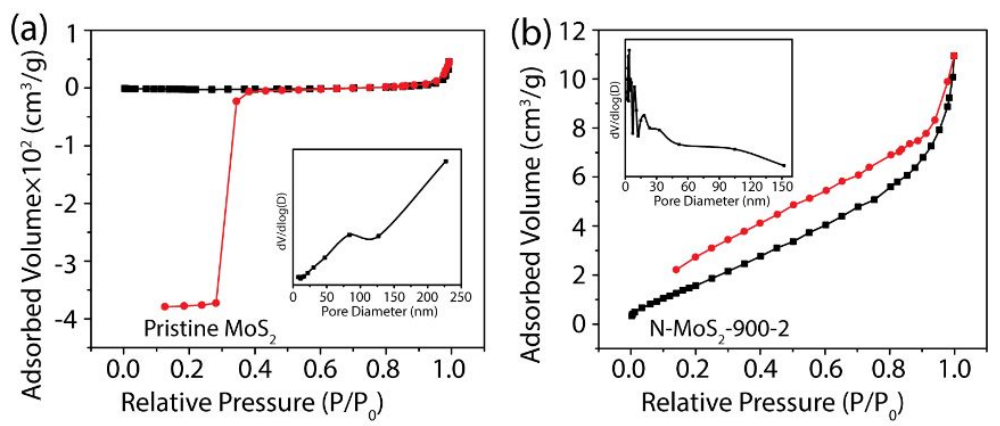

Figure S5. Nitrogen adsorption-desorption isotherms and pore size distributions of pristine $\mathrm{MoS}_{2}(\mathrm{a})$ and N-MoS$-900-2$ (b). 

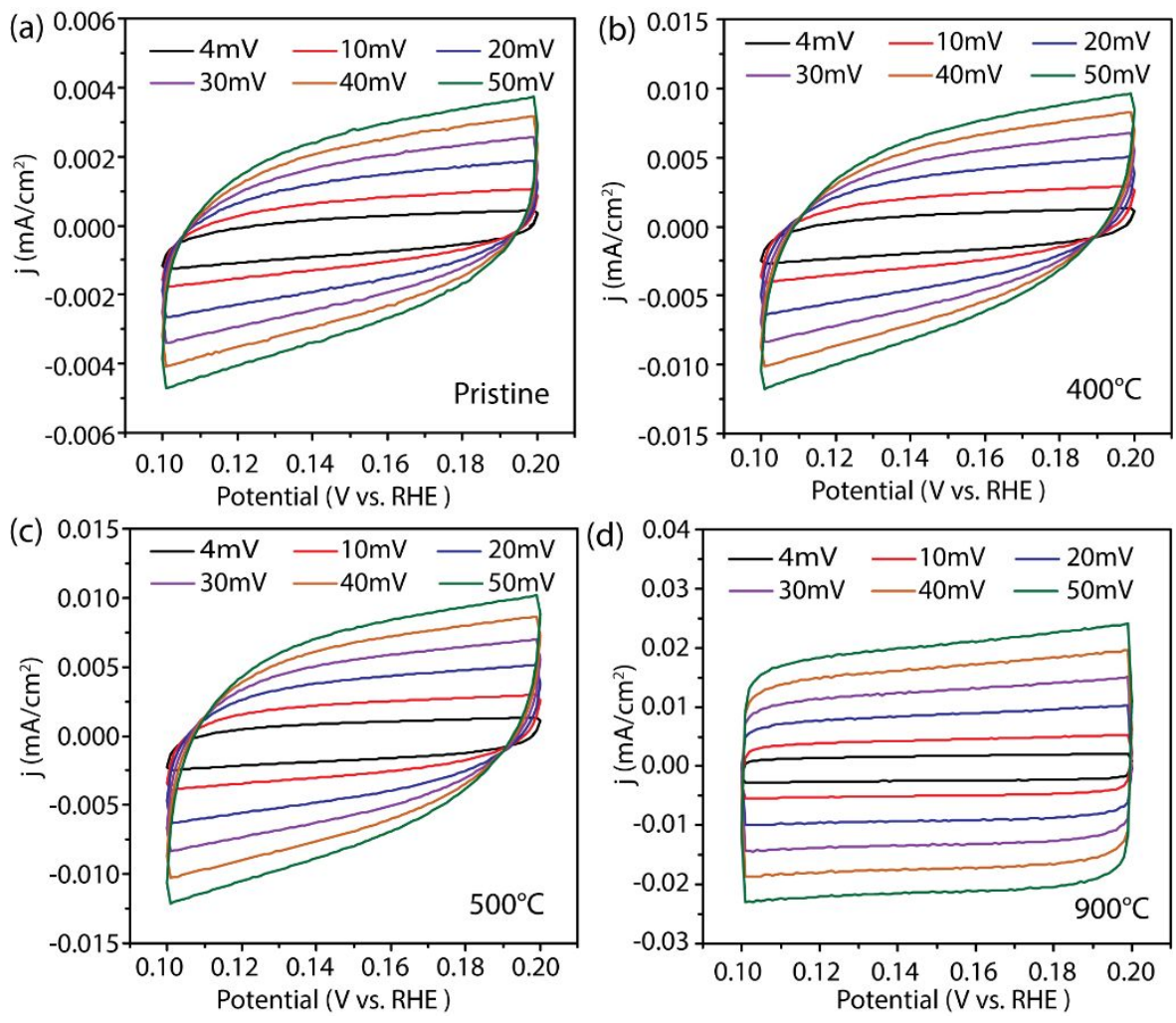

Figure S6. Electrochemical cyclic voltammograms of pristine $\mathrm{MoS}_{2}$ and $\mathrm{N}-\mathrm{MoS}_{2}$ with different activation temperatures at different scan rates $(4-50 \mathrm{mV})$.

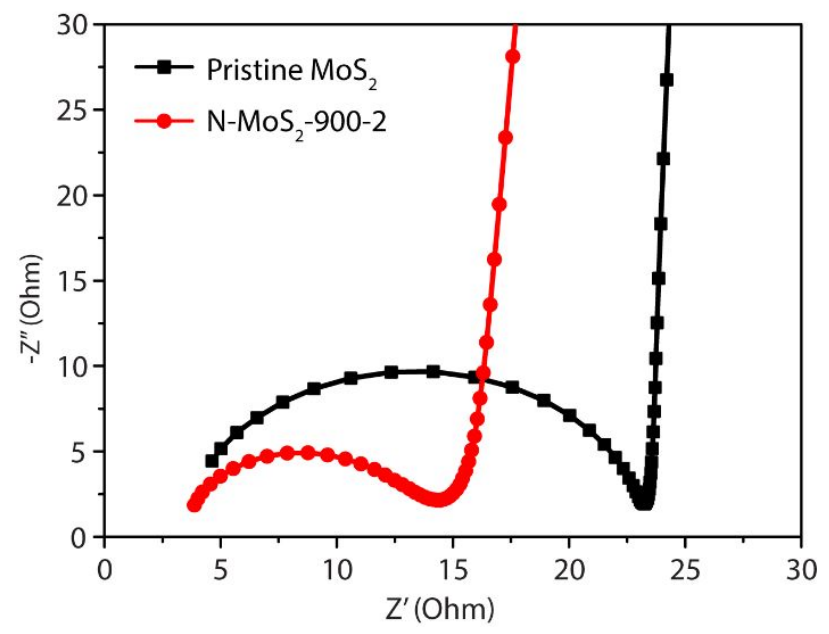


Figure S7. Electrochemical impedance spectra of pristine $\mathrm{MoS}_{2}$ and N-MoS 2 supported

on FTO from $1000 \mathrm{kHz}$ to $1 \mathrm{~Hz}$.
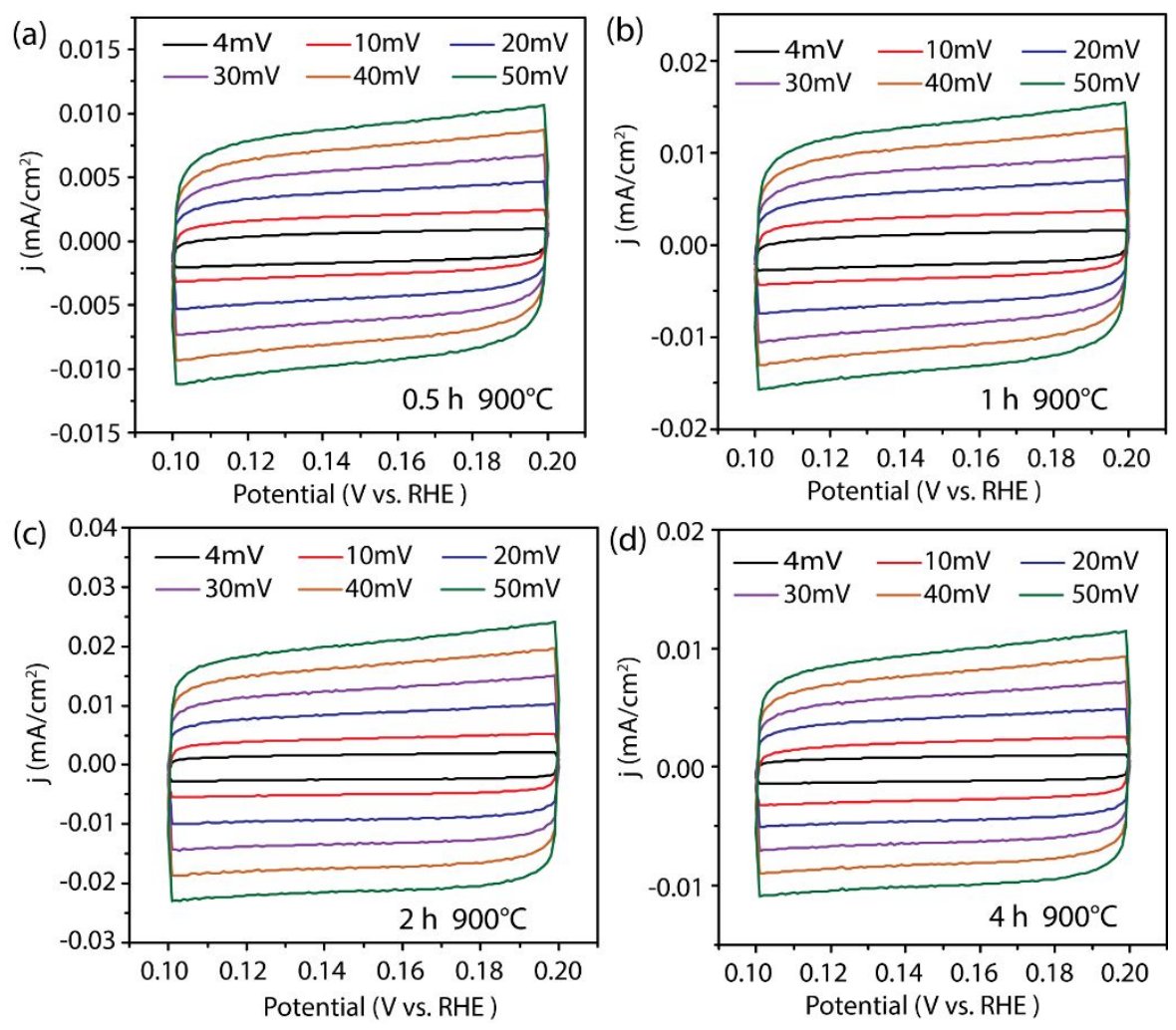

Figure S8. Electrochemical cyclic voltammograms of $\mathrm{N}-\mathrm{MoS}_{2}$ with different activation times at different scan rates $(4-50 \mathrm{mV})$. 


\section{Calculation of Electrochemical Active Surface Area (ECSA) ${ }^{1}$}

Firstly, we assume that the specific capacitance of a flat $\mathrm{MoS}_{2}$ surface is $\sim 60 \mu \mathrm{F} \mathrm{cm}{ }^{-2}$

for $1 \mathrm{~cm}^{2}$ of real surface area.

The ECSA can be calculated from the following equation:

$$
A_{E C S A}=\frac{\text { Specific capacitance }}{60 \mu \mathrm{F} \mathrm{cm}^{-2} \mathrm{~cm}_{E}^{-2} C S A}
$$

Table S1. Comparative results of surface area for our sample and other electrocatalysts

\begin{tabular}{cccccc}
\hline \multirow{3}{*}{ Materials } & Specific & & Loading & & \\
& surface area & $\mathrm{ECSA} / \mathrm{cm}^{2}$ & Amount $/ \mu$ & Geometri & $\mathrm{ECSA}_{\text {mass }} / \mathrm{S}_{\mathrm{BE}}$ \\
& $\left(\mathrm{S}_{\mathrm{BET}}\right) / \mathrm{m}^{2} \mathrm{~g}^{-1}$ & & $\mathrm{~g}$ & $\mathrm{c}$ area & $\mathrm{T}$ \\
\hline
\end{tabular}




\begin{tabular}{cccccc}
\hline $\begin{array}{c}\text { Our Work } \\
\text { Ultrathin }\end{array}$ & 7.1 & 6.65 & 20 & 0.071 & 0.33 \\
$\begin{array}{c}\mathrm{MoS}_{2} / \text { graphene }^{2} \\
\text { Mo-N/C@MoS }\end{array}{ }^{3}$ & 245 & 285 & 50 & 0.196 & 0.44 \\
$\mathrm{MoSe}_{2}$-CoSe $_{2}$ & 519.5 & 225.80 & - & 0.196 & $<0.41$ \\
Nanotubes $^{4}$ & 69.05 & 68.83 & 40 & 0.071 & 0.17 \\
\hline $\mathrm{ECSA}_{\text {mass }}$ is the value of ECSA per gram. & & & &
\end{tabular}

\section{Calculation of Per Site Turn Over Frequency (TOF) $)^{1,5,6}$}

The number of electrochemically accessible surface sites on $\mathrm{MoS}_{2}$ can be calculated

via the following equation:

$$
\frac{\# \text { of surface sites (catalyst) }}{\mathrm{cm}^{2} \text { geometric area }}=\frac{\# \text { of surface sites (flat standard) }}{\mathrm{cm}^{2} \text { geometric area }} \times \frac{C_{d l}}{60 \mu \mathrm{Fm}^{-2}}
$$

Active sites per $\mathrm{cm}^{2}$ geometric area is $1.164 \times 10^{15} \mathrm{cmreal}^{-2}$ for the flat standard. Using the equation above, the number of surface active sites for $\mathrm{N}-\mathrm{MoS}_{2}-900-2$ corresponds to $7.75 \times 10^{15}$.

Then, the following equation is employed to estimate the per site TOF:

$$
\text { TOF per site }=\frac{\# \text { Total Hydrogen Turn Over/ } \mathrm{cm}^{2} \text { geometric area }}{\# \text { Surface Sites }(\text { Catalyst }) / \mathrm{cm}^{2} \text { geometric area }}
$$


From the literature, the total number of hydrogen turn over events per geometric area at $1 \mathrm{~mA} \mathrm{~cm}-2$ is

$$
3.12 \times 10^{15} \frac{\mathrm{H}_{2} / \mathrm{s}}{\mathrm{cm}^{2}}
$$

Hence, the per site TOF of $\mathrm{MoS}_{2}-900-2$ at the current density of $14 \mathrm{~mA} \mathrm{~cm}{ }^{-2}$ is determined as follows:

$$
\mathrm{TOF}=\left(3.12 \times 10^{15} \frac{\mathrm{H}_{2} / \mathrm{s}}{\mathrm{cm}^{2}} / \frac{\mathrm{mA}}{\mathrm{cm}^{2}}\right) \times\left(14 \frac{\mathrm{mA}}{\mathrm{cm}^{2}}\right) \times\left(\frac{1 \mathrm{~cm}^{2}}{7.75 \times 10^{15} \text { surface sites }}\right)=5.6 \frac{\mathrm{H}_{2} / \mathrm{s}}{\text { surface site }}
$$

The TOF values for other samples can be calculated by same method.

Table S2. TOF values of $\mathrm{N}-\mathrm{MoS}_{2}$ samples at a potential of $0.5 \mathrm{~V}$

\begin{tabular}{lccccc}
\hline \multirow{2}{*}{ Materials } & Pristine & $\mathrm{N}-\mathrm{MoS}_{2-}$ & $\mathrm{N}-\mathrm{MoS}_{2-}$ & $\mathrm{N}-\mathrm{MoS}_{2^{-}}$ & $\mathrm{N}-\mathrm{MoS}_{2-}$ \\
& $\mathrm{MoS}_{2}$ & $900-0.5$ & $900-1$ & $900-2$ & $900-4$ \\
\hline TOF $/ \mathrm{H}_{2} \mathrm{~s}^{-1}$ & 0.34 & 2.88 & 3.92 & 5.48 & 4.00 \\
\hline
\end{tabular}




\section{References}

1. Ye, R.; del Angel-Vicente, P.; Liu, Y.; Arellano-Jimenez, M. J.; Peng, Z.; Wang, T.;

Li, Y.; Yakobson, B. I.; Wei, S.-H.; Yacaman, M. J.; et al. High-Performance Hydrogen

Evolution from $\mathrm{MoS}_{2(1-\mathrm{x})} \mathrm{P}(\mathrm{X})$ Solid Solution. Adv. Mater. 2016, 28, 1427-1432.

2. Ma, L.; Hu, Y.; Zhu, G.; Chen, R.; Chen, T.; Lu, H.; Wang, Y.; Liang, J.; Liu, H.;

Yan, C.; et al. In Situ Thermal Synthesis of Inlaid Ultrathin $\mathrm{MoS}_{2} /$ Graphene Nanosheets as Electrocatalysts for the Hydrogen Evolution Reaction. Chem. Mater. 2016, 28, 57335742.

3. Amiinu, I. S.; Pu, Z.; Liu, X.; Owusu, K. A.; Monestel, H. G. R.; Boakye, F. O.; Zhang, H.; Mu, S. Multifunctional Mo-N/C@MoS 2 Electrocatalysts for HER, OER, ORR, and Zn-Air Batteries. Adv. Funct. Mater. 2017, 27, 1702300.

4. Wang, X.; Zheng, B.; Yu, B.; Wang, B.; Hou, W.; Zhang, W.; Chen, Y. In-Situ Synthesis of Hierarchical $\mathrm{MoSe}_{2}-\mathrm{CoSe}_{2}$ Nanotubes as Efficient Electrocatalyst for 
Hydrogen Evolution Reaction in Both Acidic and Alkaline Medium. J. Mater. Chem. A 2018, 5, 1558-1566.

5. Benck, J. D.; Chen, Z.; Kuritzky, L. Y.; Forman, A. J.; Jaramillo, T. F. Amorphous Molybdenum Sulfide Catalysts for Electrochemical Hydrogen Production: Insights into the Origin of Their Catalytic Activity. ACS Catal. 2012, 2, 1916-1923.

6. Benson, J.; Li, M.; Wang, S.; Wang, P.; Papakonstantinou, P. Electrocatalytic Hydrogen Evolution Reaction on Edges of a Few Layer Molybdenum Disulfide Nanodots. ACS Appl. Mater. Interfaces 2015, 7, 14113-14122. 